\title{
Development of Sarcoidosis during Etanercept Therapy
}

\author{
Takashi Ishiguro ${ }^{1}$, Noboru Takayanagi ${ }^{1}$, Kazuyoshi Kurashima ${ }^{1}$, Aya Matsushita ${ }^{1}$, \\ Keiji Harasawa ${ }^{1}$, Koichiro Yoneda ${ }^{1}$, Noriko Tsuchiya ${ }^{1}$, Yousuke Miyahara ${ }^{1}$, \\ Shozaburo Yamaguchi ${ }^{1}$, Ryozo Yano ${ }^{1}$, Daidou Tokunaga ${ }^{1}$, Hiroo Saito ${ }^{1}$, Mikio Ubukata ${ }^{1}$, \\ Tsutomu Yanagisawa ${ }^{1}$, Yutaka Sugita ${ }^{1}$ and Yoshinori Kawabata ${ }^{2}$
}

\begin{abstract}
This report describes a 65-year-old woman who developed granulomatous lesions consistent with sarcoidosis during etanercept therapy for rheumatoid arthritis. Hilar and mediastinal lymphadenopathy and multiple nodules in both lung fields developed 21 months after administration of etanercept. Noncaseating epithelioid cell granulomas consistent with sarcoidosis were detected in a lung biopsy specimen and in the parietal pleura obtained via thoracotomy. Diseases showing similar histologic changes were excluded, and a diagnosis of sarcoidosis was made. Etanercept was discontinued, which resulted in symptomatic relief, improvement of oxygenation and radiologic findings. There is substantial evidence of tumor necrosis factor-alpha involvement in the induction and maintenance of granuloma formation; however, we should keep in mind that granulomatous disease, such as sarcoidosis, can develop during treatment with a tumor necrosis factor-alpha blocking agent, such as etanercept.
\end{abstract}

Key words: sarcoidosis, etanercept, rheumatoid arthritis, TNF- $\alpha$

(Inter Med 47: 1021-1025, 2008)

(DOI: 10.2169/internalmedicine.47.0602)

\section{Introduction}

Tumor necrosis factor-alpha (TNF- $\alpha)$ blockade with the soluble receptor fusion protein etanercept and the monoclonal antibody infliximab has recently been shown to be effective in the treatment of rheumatoid arthritis (RA). Because TNF- $\alpha$ is a key cytokine involved in granuloma formation, TNF- $\alpha$ blockade, which suppresses granuloma formation, has been suggested to be a potential treatment for sarcoidosis. Certainly, etanercept has been reported to be used successfully in some cases to treat sarcoidosis $(1,2)$. In this report, however, we describe a patient who developed sarcoidosis with lung involvement during etanercept therapy for RA.

\section{Case Report}

A 65-year-old woman was admitted to our hospital in
May 2007 for hilar lymphadenopathy and nodules in both lung fields. RA had been diagnosed in 2001 and was first treated with aurothiomalate sodium, methotrexate, and lowdose prednisolone ( $2.5 \mathrm{mg}$ daily). Although the patient experienced slight improvement in morning stiffness and dactylitis with this treatment, gastrointestinal symptoms developed, which improved after the withdrawal of the methotrexate. In September 2005, chest radiography and computed tomography (CT) of the chest showed no abnormal findings, and etanercept therapy was started at $25 \mathrm{mg}$ twice weekly. Within 2 months, the patient noted a marked improvement of her symptoms. However, she complained of general fatigue in April 2007. Chest radiography in May 2007 showed nodules in the bilateral lung fields with hilar lymphadenopathy (Fig. 1a). CT of the chest showed mediastinal and hilar lymphadenopathy (Fig. 1b), and nodules with perilymphatic distribution in both lung fields (Fig. 1c). The patient was admitted to our hospital for further examination. Upon admission, no skin lesions were found and chest auscultation re-

${ }^{1}$ Department of Respiratory Medicine, Saitama Cardiovascular and Respiratory Center, Kumagaya and ${ }^{2}$ Division of Pathology, Saitama Cardiovascular and Respiratory Center, Kumagaya

Received for publication September 12, 2007; Accepted for publication December 2, 2007

Correspondence to Dr. Takashi Ishiguro, ishiguro@med3.m.kanazawa-u.ac.jp 

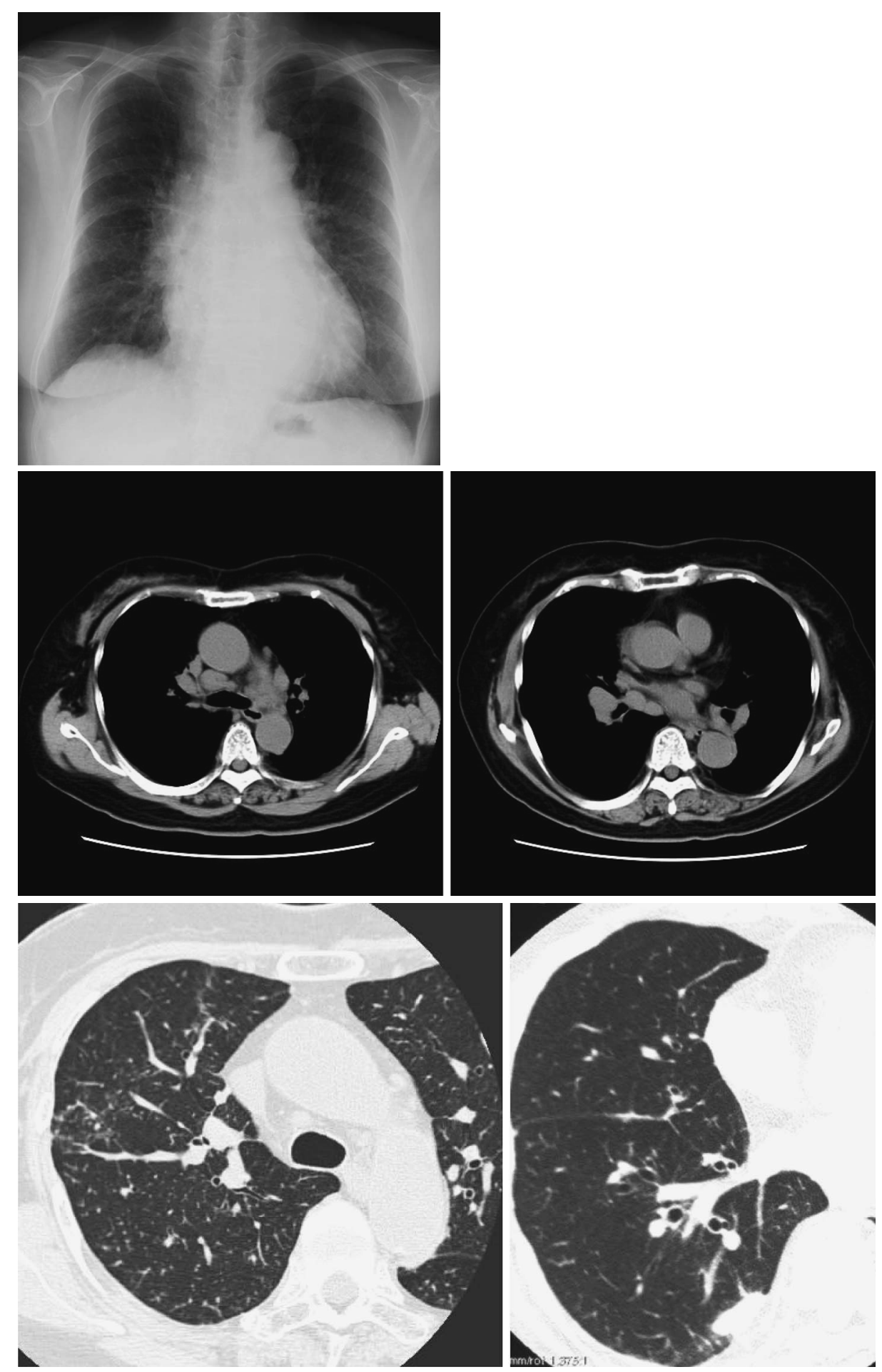

Figure 1. Chest radiography and Computed Tomography (CT) on admission. Chest radiography on admission (a) showed bilateral and diffuse small nodular lesions with hilar lymphadenopathy. Chest computed tomography (CT) showed mediastinal and hilar lymphadenopathy (b), diffuse and small nodular lesions in the lung field and interlober pleura, and a tuberous lesion in the lower lobe (c).

vealed no abnormal sounds. There were no findings suggesting either ocular or neural sarcoidosis. The results of an electrocardiogram and echocardiography were normal. The reaction to a purified protein derivative skin test was negative. A blood gas analysis while the patient was breathing room air in the sitting position showed $\mathrm{pH} 7.41$, a partial pressure of oxygen $\left(\mathrm{PaO}_{2}\right)$ 62.5 Torr, carbon dioxide $\left(\mathrm{PaCO}_{2}\right)$ 40.3 Torr, and bicarbonate $25.0 \mathrm{mmol} / \mathrm{l}$. Respiratory function tests revealed almost normal findings except for a slight decrease of vital capacity at $2.901(77.6 \%$, \% predicted), forced vital capacity 2.901 (77.6\%, \% predicted), and diffusing capacity of carbon monoxide $15.13 \mathrm{ml} / \mathrm{min} /$ 


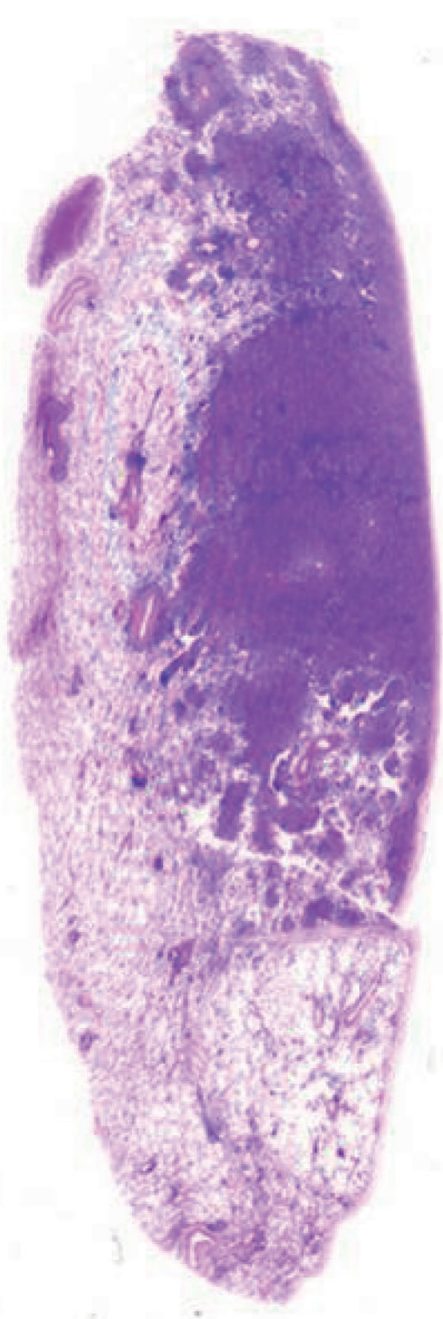

a
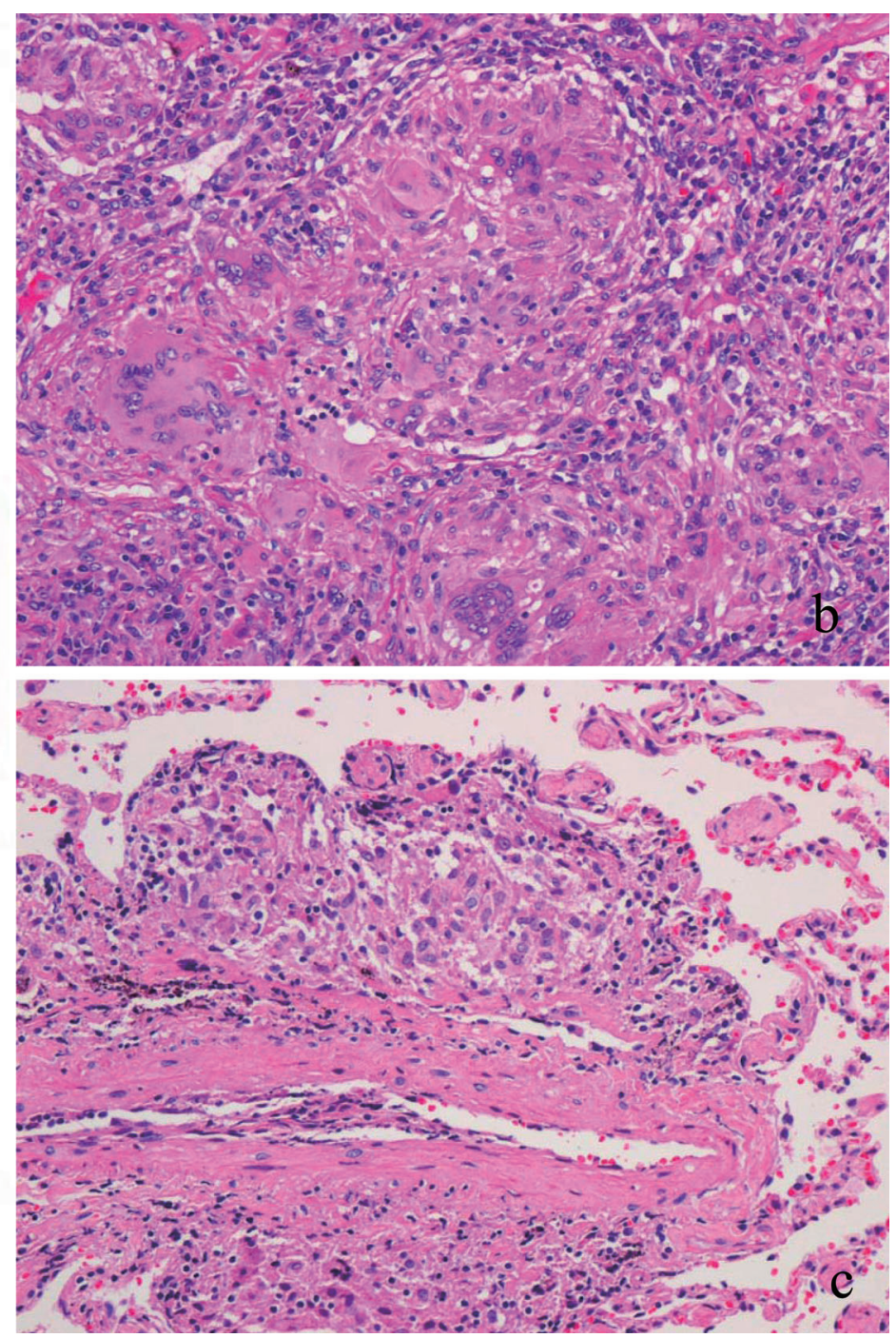

Figure 2. a. Panoramatic view showing the subpleural irregularly difined nodular lesion and many granulomas along lymphatic routes. b. The nodular lesion was composed of confluent epithelioid cell granulomas without caseous necrosis. ${ }^{*} 200$. c. Epithelioid cell granulomas located the vascular wall. *200. Each was stained by Hematoxyline and Eosin Staining.

Torr $(91.6 \%, \%$ predicted). Laboratory values including peripheral blood cell counts and biochemistry were within normal limits. The erythrocyte sedimentation rate was $30 \mathrm{~mm} /$ hr. The serum C-reactive protein concentration was $0.38 \mathrm{mg} /$ dL. Serum angiotensin converting enzyme (ACE) was not elevated (21.3 U/L. normal range, 7.0-25.0 U/L) and serum lysozyme concentration was slightly elevated $(8.3 \mu \mathrm{g} / \mathrm{mL}$. normal range, $2.7-7.5 \mu \mathrm{g} / \mathrm{mL}$ ). The soluble interleukin (IL)-2 receptor concentration was $998 \mathrm{U} / \mathrm{mL}$, and the serum IL-6 concentration was below $8 \mathrm{pg} / \mathrm{mL}$. The serum interferon- $\gamma$ concentration was below $0.4 \mathrm{IU} / \mathrm{mL}$. Gallium 67 scintigraphy showed an increased uptake in the paratracheal and hilar lymph nodes and both lung fields. Bronchoalveolar lavage of the right middle lobe (B4b) was performed. The total cell count was $10.3 \times 10^{5}$ cells $/ \mathrm{ml}$, with $74.2 \%$ macrophages, $0.2 \%$ neutrophils, $0.4 \%$ eosinophils, and $25.2 \%$ lymphocytes. The CD4/CD8 ratio was elevated at 6.6. No granulomas or specific changes were found in a transbronchial lung biopsy specimen; therefore, lung biopsy via video-assisted thoracic surgery was performed for the differential diagnosis of pulmonary disease. Histopathologic study of the superior segment of the right lower lobe, in which a large nodule was detected with CT, showed numerous confluent noncaseating epithelioid cell granulomas (ECGs) and tiny noncaseating ECGs in the lung and parietal pleura (Fig. 2a, b). The ECGs were mainly distributed along the lymphatic pathways. The results of tissue staining and a culture for bacteria, fungus, and mycobacterium, as well as a polymerase chain reaction analysis for Mycobacterium tuberculosis and Mycobacterium avium complex were all negative. The diagnosis of sarcoidosis was made on the basis of the American Thoracic Society/European Respiratory Society/ World Association of Sarcoidosis and Other Granulomatous Disorders criteria (3). Because of a possible relation between the development of sarcoidosis and treatment with etanercept, and because of the existence of case reports de- 
Table 1. Sarcoid-Like Granulomatous Reactions Associated with TNF-Blockade

\begin{tabular}{|c|c|c|c|c|c|c|}
\hline Author (year) & Ref & Age, Sex & \multicolumn{2}{|c|}{ TNF-blockade } & $\begin{array}{l}\text { Time to } \\
\text { reaction }\end{array}$ & Pathological features \\
\hline Peno-Green et al (2002) & 8 & $50, \mathrm{~F}$ & RA & Etanercept & 2 & Noncaseating granulomas in lungs and skin \\
\hline Hashkes et al (2003) & 9 & 7, M & JRA & Etanercept & 1 & Noncaseating granulomas in the liver \\
\hline Hubscher et al (2003) & 10 & $41, \mathrm{~F}$ & RA & Etanercept & 19 & $\begin{array}{l}\text { Necrotizing area surounded by a palisade of } \\
\text { histiocytes and chronic inflammatory cells }\end{array}$ \\
\hline Vavricka et al (2003) & 14 & $73, \mathrm{M}$ & RA & Adalimumab & 12 & $\begin{array}{l}\text { Necrotizing granulomatous inflammation with } \\
\text { mutinucleated giant cells }\end{array}$ \\
\hline Gonzalez-Lopez et al(2006) & 11 & $70, \mathrm{M}$ & AS & Etanercept & 21 & Noncaseating granulomas in skins \\
\hline O'Shea et al (2006) & 16 & $34, \mathrm{M}$ & AS & infliximab & 60 & Nonnecrotizing granulomas in lungs, lymph nodes \\
\hline Vershueren et al (2007) & 12 & $53, \mathrm{~F}$ & RA & Etanercept & 6 & Noncaseating granulomas in mediastinal lymph nodes \\
\hline Vershueren et al (2007) & 12 & $46, \mathrm{~F}$ & RA & Etanercept & 12 & Noncaseating granulomas in mediastinal lymph nodes \\
\hline Almodovar et al (2007) & 15 & $34, \mathrm{M}$ & AS & infliximab & 12 & Noncaseating granulomas in lungs \\
\hline Kudrin A et al (2007) & 13 & $52, \mathrm{~F}$ & RA & Etanercept & 18 & Noncaseating granulomas in the lung \\
\hline The present case & - & $57, \mathrm{~F}$ & RA & Etanercept & 25 & Noncaseating granulomas in the lung \\
\hline
\end{tabular}

\begin{tabular}{|c|c|}
\hline Author (year) & Treatment/Outcome \\
\hline $\begin{array}{l}\text { Peno-Green et al (2002) } \\
\text { (200 }\end{array}$ & Stabilization after etanercept withdrawal/Resolution with prednisone \\
\hline Hashkes et al (2003) & Etanercept withdrawal/Slight improvement with methotrexate \\
\hline Hubscher et al (2003) & Etanercept withdrawal/Outcome not specified \\
\hline Vavricka et al (2003) & Adalimumab withdrawal/persistence despite antituberculous therapy \\
\hline Gonzalez-Lopez et al(2006) & Etanercept withdrawal/Resolution 2 months after withdrawal \\
\hline O'Shea et al (2006) & Symptomatic and radiological improvement with prednisolone \\
\hline Vershueren et al (2007) & Etanercept withdrawal/Complete remission with methylprednisolone \\
\hline Vershueren et al (2007) & Etanercept withdrawal/Complete remission with no additional therapy \\
\hline Almodovar et al (2007) & Infliximab withdrawal/Complete remission with no additional therapy \\
\hline Kudrin A et al (2007) & Symptomatic and radiological improvement with prednisolone \\
\hline The present case & Etanercept withdrawal/Improvement \\
\hline
\end{tabular}

scribing that sarcoidosis developed during etanercept therapy with an improvement after the withdrawal of the drug $(4,5)$, etanercept therapy was therefore stopped. This resulted in a symptomatic relief and radiologic improvement within 2 months. Blood gas analysis improved $(\mathrm{pH} 7.419$, a partial pressure of oxygen $\left(\mathrm{PaO}_{2}\right) 71.2$ Torr, carbon dioxide (PaCO 2) 40.1 Torr, and bicarbonate $25.5 \mathrm{mmol} / \mathrm{l})$. The patient was discharged and has been in good health without any symptoms. She has been followed-up regularly on an outpatient basis.

\section{Discussion}

The recent progress in the therapy for RA has been remarkable, and some novel biological agents have helped us to improve the control of RA which had previously been refractory to the usual treatment. Simultaneously, it has been reported that various diseases or side effects, including respiratory tract infections, sometimes develop during the administration of these agents.

To date, there are three TNF- $\alpha$-blocking agents currently available: etanercept (a soluble TNF- $\alpha$ receptor fusion protein), infliximab (a humanized mouse monoclonal antibody), and adalimumab (a fully human monoclonal antibody). It has been reported that respiratory tract infections, especially mycobacterial and fungal infections which can induce granuloma formation, develop during the administration of
TNF- $\alpha$ blockade. In the present case, however, the results of a culture as well as polymerase chain reaction analysis for infection were all negative, and the evident infectious agents were excluded.

Sarcoidosis is considered to be a systemic, granulomatous, and immune-mediated inflammatory disorder. There is evidence that inflammation and granuloma formation occur in a genetically susceptible host as a result of a Th1-type immune response. This is characterized by large numbers of activated macrophages and CD4+ T lymphocytes. Although a large variety of cytokines are produced under these conditions, TNF- $\alpha$ as well as interferon- $\gamma$ (IFN- $\gamma$ ), IL-12, and IL18 play critical roles in driving the Th1 commitment in the course of the granulomatous process $(6,7)$. Therefore, the development of granulomatous reactions during a TNF- $\alpha$ blockade in this case seems paradoxical. However, there have been some reports of sarcoid-like granuloma/sarcoidosis development during TNF- $\alpha$-blockade for RA and for ankylosing spondylitis (8-16) (Table 1).

In this case, sarcoidosis improved after the withdrawal of etanercept, thus suggesting a relation between the development of sarcoidosis and etanercept administration. It is not known whether etanercept can cause sarcoidosis, but two hypotheses of pathogenic mechanism can be raised. One is the immunosuppressant effect of biological therapies that could favor the mediation of infectious agents in the development of sarcoidosis because some microorganisms such as 
Propionibacterium acnes and Propionibacterium granulosum have been identified as possible etiologic agents (17). The other is the involvement of other cytokines that could play a crucial role in granuloma formation under treatment with TNF- $\alpha$-blockade. There are some reports supporting the latter hypothesis. It is known that patients treated with etanercept show a significant increase in the percentage of $\mathrm{T}$ cells expressing IFN- $\gamma(18)$, which is one of the lymphokines most relevant to the generation of granulomas and is known to be significantly increased in the serum of patients with sarcoidosis compared controls (19). In addition, some investigators reported that pulmonary sarcoidosis worsened in 11 of 16 cases after etanercept administration (4).

The relation between infliximab and granulomatous formation remains controversial. As shown in Table 1, 2 cases who developed sarcoid-like granulomas/sarcoidosis during the infliximab therapy have been reported $(14,15)$, but these cases were contradictory to previous published studies that report an improvement in sarcoid manifestation by infliximab therapy $(20,21)$. There are differences in the kinetics, the binding avidities for soluble versus transmembrane TNF$\alpha$ (12), and the ability to inhibit IFN- $\gamma$ production (18) between etanercept and other types of TNF- $\alpha$ blockade. These observations may explain the different efficacy of these agents for sarcoidosis, or the frequency of the development of sarcoidosis under TNF- $\alpha$ blockade; however, data are limited, and further studies are needed to clarify these matters.

In summary, this report describes a patient with RA who developed sarcoidosis during etanercept therapy. We should keep in mind that a granulomatous disease, such as sarcoidosis, can develop during TNF- $\alpha$-blockade therapy.

\section{References}

1. Khanna D, Liebling MR, Louie JS. Etanercept ameliorates sarcoidosis arthritis and skin disease. J Rheumatol 30: 1864-1867, 2003.

2. Hobbs K. Chronic sarcoid artheitis treated with intraarticular etanercept [letter]. Arthritis Rheum 52: 987-988, 2005.

3. Hunninghake GW, Costabel U, Ando M, et al. ATS/ERS/WASOG statement on sarcoidosis: American Thoracic Society/European Respiratory Society/World Association of Sarcoidosis and other Granulomatous Disorders. Sarcoidosis Vasc Diffuse Lung Dis 16: 149-173, 1999.

4. Utz JP, Limper AH, Kalra S, et al. Etanercept for the treatment of stage II and III progressive pulmonary sarcoidosis. Chest 124: 177-185, 2003

5. Phillips K, Weinblatt M. Granulomatous lung disease occurring during etanercept treatment. Arthritis Rheum 53: 618-620, 2005.

6. Kindler V, Sappino A, Grau GE, Piguet PF, Vassalli P. The inducing role of tumor necrosis factor in the development of bactericidal granulomas during BCG infection. Cell 56: 731-740, 1989.

7. Bost TW, Riches DW, Schumacher B, et al. Alveolar macrophages from patients with beryllium disease and sarcoidosis express increased levels of mRNA for tumor necrosis factor- $\alpha$ and interleukin-6 but not interleukin-1 $\beta$. Am J Respir Cell Mol Biol 10: 506-513, 1994.

8. Peno-Green L, Lluberas G, Kingsley T, Brantley S. Lung injury linked to etanercept treatment. Arthritis Rheum 48: 2077-2078, 2003.

9. Hashkes PJ, Shajrawi I. Sarcoid-related uveitis occurring during etanercept therapy. Clin Exp Rheum 21: 645-646, 2003.

10. Hubscher O, Re R, Iotti R. Pulmonary rheumatoid nodules in an etanercept-treated patient [letter]. Arthritis Rheum 48: 2077-2078, 2003.

11. Gonzalez-Lopez MA, Blanco R, Gonzalez-Vela MC, FernandezLlaca H, Rodriguez V. Development of sarcoidosis during etaner- cept therapy. Arthritis Rheum 55: 817-820, 2006.

12. Verschueren K, Van Essche E, Patrick V, Taelman V, Rene. Development of sarcoidosis in etanercept treated rheumatoid arthritis patients. Clin Rheumatol 6: 1969-1971, 2007.

13. Kudrin A, Chilvars ER, Ginawi A, et al. Sarcoid-like granulomatous disease following etanercept treatment for rheumatoid arthritis. J Rheum 34: 648-649, 2007.

14. Vavricka SR, Wettstein T, Speich R, Gaspert A, Bachli EB. Pulmonary granulomas after tumor necrosis factor $\alpha$ antagonist therapy. Thorax 58: 278-279, 2003.

15. Almodovar R, Izquierdo $M$, Zarco $P$, Javier Quiros $F$, Mazzucchelli R, Steen B. Pulmonary sarcoidosis in a patient with ankylosing spondylitis treated with infliximab. Clin Exp Rheumatol 25: 99-101, 2007.

16. O'Shea FD, Marras TK, Inman RD. Pulmonary sarcoidosis developing during infliximab therapy. Arthritis Rheum 55: 978-981, 2006.

17. Eishi Y, Suga M, Ishige I, et al. Quantitative analysis of mycobacterial and propionibacterial DNA in lymph nodes of Japanese and European patients with sarcoidosis. J Clin Microbiol 40: 198-204, 2002.

18. Furst DE, Wallis R, Broder M, Beenhauwer DO. Tumor necrosis factor antagonist: different kinetics and/or mechanisms of action may explain differences in the risk for developing granulomatous infection. Semin Arthritis Rheum 36: 159-167, 2006.

19. Prior C, Haslam PL. Increased levels of serum interferon-gamma I pulmonary sarcoidosis and relationship with response to corticosteroid therapy. Am Rev Respir Dis 143: 53-60, 1991.

20. Doty JD, Mazur JE, Judson MA. Treatment of sarcoidosis with infliximab. Chest 127: 1064-1071, 2005.

21. Keystone EC. The utility of tumor necrosis factor blockade in orphan diseases. Ann Rheum Dis 63 (Suppl 2): ii79-ii83, 2004.

(C) 2008 The Japanese Society of Internal Medicine

http://www.naika.or.jp/imindex.html 